\title{
SIMULATIONS AND EXPERIMENTS OF HIGH-SPEED STEALTH WALKING BASED ON A REALISTIC CONTROL APPROACH
}

\author{
FUMIHIKO ASANO, MASATSUGU NISHIHARA AND MASASHI KAWAZOE \\ School of Information Science, Japan Advanced Institute of Science and Technology, \\ 1-1 Asahidai, Nomi, Ishikawa 923-1292, Japan \\ E-mail: \{fasano,s1820026,s1810045\}@jaist.ac.jp
}

\begin{abstract}
This paper proposes a realistic control method for generating a high-speed stealth walking gait of an underactuated rimless wheel (URW) with an elastic element taking the hardware characteristics into account. First, we introduce the experimental URW machine and its mathematical model. Second, we propose a method for driving the relative joint according to the acceleration command signal of a simple sinusoidal wave. In this approach, the resultant relative-angle trajectory also becomes a simple sinusoidal wave, and the stance-leg motion that behaves as zero dynamics is also able to satisfy the target condition necessary for stealth walking. Third, we extend the method developed through investigation of the linearized model to the nonlinear model, and numerically confirm the validity. Finally, we report some basic experimental results of the prototype URW machine.
\end{abstract}

\section{Introduction}

In general, point-footed limit-cycle walkers have one degree of underactuation at ankle or ground-contact point, and their relative joint angles are chosen as the control output for generating an efficient walking motion. ${ }^{1}$ In this approach, the absolute angle of the stanceleg behaves as hybrid zero dynamics (HZD), and the main problem is how to guarantee or understand the stability of HZD.

The authors have proposed a different approach to generation of an underactuated walking motion aiming at adaptation to various ground condition; generation of a careful walking pattern considering rough terrain. ${ }^{2-4}$ Unlike limit-cycle walking, ${ }^{1}$ in this approach the absolute stance-leg angle is chosen as one of the control outputs, and is strictly and preferentially controlled so that the fore foot can land on the ground stealthily without causing the loss of kinetic energy. ${ }^{5,6}$ From the practical point of view, however, it is not easy to precisely measure the absolute stance-leg angle in real-time, and it is most realistic to directly drive the relative joint angle to which the motor is attached by utilizing the dedicated positional controller. As for the desired trajectory, it is manageable to use a sinusoidal wave in which only the target step period and amplitude need to be adjusted.

Based on the observation, in this paper we apply the control technique for limit-cycle walkers to a real stealth walking machine shown in Fig. 1 left. This machine consists of two identical cross-shaped frames and a disk-shaped reaction wheel. The mounting angle between the two cross-shaped frames can be adjusted for generating an asymmetric walking gait. ${ }^{7}$ The reaction wheel is connected to the rimless wheel incorporating a maxon EC motor and a spiral spring as shown in Fig. 1 center. In stealth walking, the stance leg is accelerated during the first half of the single-limb support phase and decelerated during the second half. Although the loss of kinetic energy at impact can be avoided, deceleration during the second half causes gait inefficiency; there is a trade-off. Therefore, it should be considered to add an elastic element between the stance leg and reaction wheel. By the effect of the spiral spring, our machine can reduce the load of the EC motor, and improvement of energy efficiency can be expected. The EC motor is controlled by utilizing the dedicated digital positional controller EPOS2 in real-time, and a simple PD control is applied for tracking the desired 

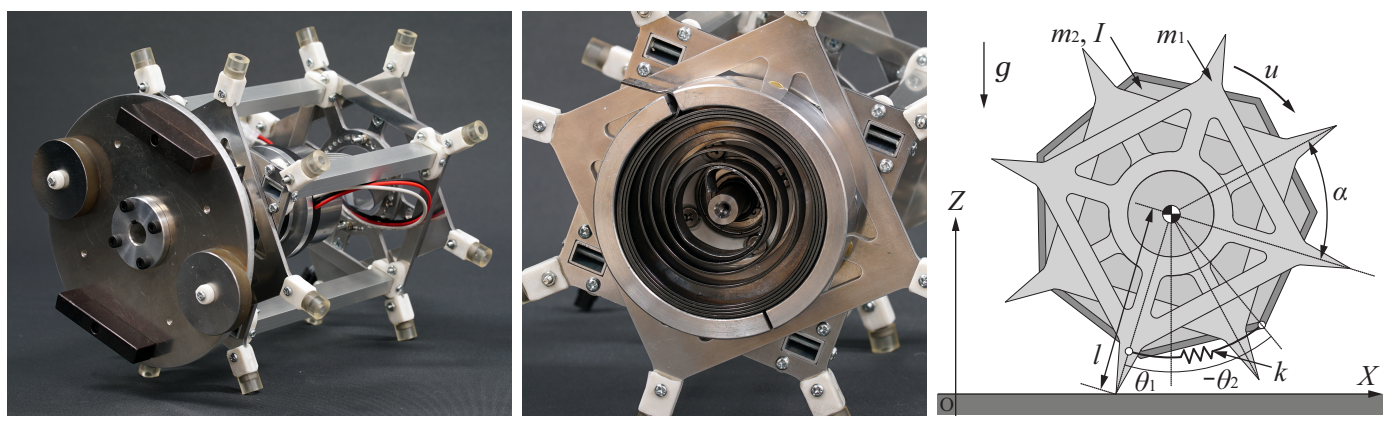

Fig. 1. Prototype experimental machine of eight-legged underactuated rimless wheel with spiral spring and its mathematical model

sinusoidal trajectory. The proposed control method also makes it possible to generate a smooth control torque.

\section{Underactuated Rimless Wheel and Control Law}

\subsection{Model assumptions and equation of motion}

Fig. 1 right shows the model of the eight-legged URW that consists of two identical crossshaped frames and a reaction wheel. ${ }^{8}$ An elastic element with elastic modulus of $k[\mathrm{~N} \cdot \mathrm{m} / \mathrm{rad}]$ is installed between the cross-shaped frame and reaction wheel so that the elastic force becomes zero where $\theta_{1}=\theta_{2}$. The reaction wheel is therefore essentially stable at $\theta_{2}=\theta_{1}$. In this model, $m\left(=m_{1}+m_{2}\right)[\mathrm{kg}]$ is the total mass of the URW; $l[\mathrm{~m}]$ is the stance-leg length; $I\left[\mathrm{~kg} \cdot \mathrm{m}^{2}\right]$ is the inertia moment of the reaction wheel; $\alpha[\mathrm{rad}]$ is the relative angle between two adjacent leg frames and is equal to $\pi / 4[\mathrm{rad}] ; u[\mathrm{~N} \cdot \mathrm{m}]$ is the control torque exerted between the stance leg and reaction wheel.

The $\theta_{1}$ and $\theta_{2}$ are the angular position of the stance leg and reaction wheel with respect to vertical. Let $\boldsymbol{\theta}=\left[\begin{array}{ll}\theta_{1} & \theta_{2}\end{array}\right]^{\mathrm{T}}$ be the generalized coordinate vector. The robot equation of motion then becomes

$$
\boldsymbol{M} \ddot{\boldsymbol{\theta}}+\boldsymbol{h}=\boldsymbol{S} u
$$

where terms $\boldsymbol{M}, \boldsymbol{h}$ and $\boldsymbol{S}$ are detailed as

$$
\boldsymbol{M}=\left[\begin{array}{cc}
m l^{2} & 0 \\
0 & I
\end{array}\right], \quad \boldsymbol{h}=\left[\begin{array}{c}
-m g l \sin \theta_{1}+k\left(\theta_{1}-\theta_{2}\right) \\
-k\left(\theta_{1}-\theta_{2}\right)
\end{array}\right], \quad \boldsymbol{S}=\left[\begin{array}{c}
1 \\
-1
\end{array}\right] .
$$

We assume that the fore foot lands on the ground stealthily without causing the loss of kinetic energy according to the control law described later, and that the stance-leg exchange is completed smoothly. The angular velocities therefore do not change at this instant, that is, $\dot{\boldsymbol{\theta}}^{-}=\dot{\boldsymbol{\theta}}^{+}$holds where the superscripts "-" and "+" denote immediately before and immediately after stance-leg exchange. The stance leg and reaction wheel are controlled to rotate clockwise by $\alpha$ [rad] during every single-support phase, and their angular positions should be reset to $\boldsymbol{\theta}^{+}=\boldsymbol{\theta}^{-}-\left[\begin{array}{ll}\alpha & \alpha\end{array}\right]^{\mathrm{T}}$. The generated motions of the stance leg and reaction wheel therefore include state jumps and behave as HZD, but they are not affected by collision dynamics.

\subsection{Control law}

In stealth walking, the stance-leg motion must be controlled strictly and preferentially so that the fore foot can land on the ground stealthily without causing the loss of kinetic 
energy. In this paper, however, we propose a different approach to stealth walking taking the hardware into account described below.

We choose the control output as $y:=\boldsymbol{S}^{\mathrm{T}} \boldsymbol{\theta}=\theta_{1}-\theta_{2}$ instead of $\theta_{1}$ because the EC motor is installed between the leg frame and reaction wheel. The control input for achieving $\ddot{y}=v$ can be determined as

$$
u=\frac{v+\boldsymbol{S}^{\mathrm{T}} \boldsymbol{M}^{-1} \boldsymbol{h}}{\boldsymbol{S}^{\mathrm{T}} \boldsymbol{M}^{-1} \boldsymbol{S}}=\frac{m l^{2} I}{m l^{2}+I}\left(v-\omega^{2} \sin \theta_{1}\right)+k y,
$$

where $\omega:=\sqrt{g / l}[\mathrm{rad} / \mathrm{s}]$. As discussed in Ref. 9, the $y$ can be controlled to the desired terminal value according to the methods of discrete-time output deadbeat control (DODC) or continuous-time output deadbeat control (CODC) laws, and a stable limit-cycle gait can be generated consequently. DODC is a minimum-time or bang-bang control, whereas CODC is an averaging method of DODC by using a linear function of time. In this paper, however, we consider the following command signal, $v=v(t)$, for angular acceleration.

$$
v(t)=A_{m} \sin \left(\frac{2 \pi t}{T}\right)
$$

We assume that the time parameter $t[\mathrm{~s}]$ is reset to zero at every instant of stance-leg exchange. This $v(t)(=\ddot{y})$ implies that the EC motor can exert a smooth control torque unlike DODC. According to this $v(t)$, the resultant output trajectory, $y$, also becomes a simple sinusoidal wave as described later. This approach, DODC and CODC have in common that they accelerate in the first half of cycle and decelerate in the second half.

\section{Analysis of Linearized Model}

\subsection{Derivation of approximate analytical solution for target system parameters}

The linearized equation of motion becomes

$$
\boldsymbol{M} \ddot{\boldsymbol{\theta}}+\boldsymbol{G \theta}=\boldsymbol{S} u,
$$

where

$$
\boldsymbol{G}=\left[\begin{array}{cc}
-m g l+k & -k \\
-k & k
\end{array}\right] .
$$

The control input for achieving $\ddot{y}=v(t)$ in the linearized model is then determined as

$$
u=\frac{v(t)+\boldsymbol{S}^{\mathrm{T}} \boldsymbol{M}^{-1} \boldsymbol{G} \boldsymbol{\theta}}{\boldsymbol{S}^{\mathrm{T}} \boldsymbol{M}^{-1} \boldsymbol{S}}=\frac{m l^{2} I}{m l^{2}+I}\left(v(t)-\omega^{2} \theta_{1}\right)+k y .
$$

By substituting this control input into Eq. (5) and arranging it, we can obtain the state-space realization of the controlled linearized equation of motion as

$$
\dot{\boldsymbol{x}}=\boldsymbol{A} \boldsymbol{x}+\boldsymbol{B} v(t),
$$

where $\boldsymbol{x}=\left[\boldsymbol{\theta}^{\mathrm{T}} \dot{\boldsymbol{\theta}}^{\mathrm{T}}\right]^{\mathrm{T}}$ and

$$
\begin{aligned}
& \boldsymbol{A}=\left[\begin{array}{cc}
\mathbf{0}_{2 \times 2} & \boldsymbol{I}_{2} \\
-\boldsymbol{M}^{-1}\left(\boldsymbol{I}_{2}-\frac{\boldsymbol{S} \boldsymbol{S}^{\mathrm{T}} \boldsymbol{M}^{-1}}{\boldsymbol{S}^{\mathrm{T}} \boldsymbol{M}^{-1} \boldsymbol{S}}\right) \boldsymbol{G} & \mathbf{0}_{2 \times 2}
\end{array}\right]=\left[\begin{array}{cccc}
0 & 0 & 1 & 0 \\
0 & 0 & 0 & 1 \\
\frac{m g l}{m l^{2}+I} & 0 & 0 & 0 \\
\frac{m g l}{m l^{2}+1} & 0 & 0 & 0
\end{array}\right], \\
& \boldsymbol{B}=\left[\begin{array}{c}
\mathbf{0}_{2 \times 1} \\
\frac{\boldsymbol{M}^{-1} \boldsymbol{S}}{\boldsymbol{S}^{\mathrm{T}} \boldsymbol{M}^{-1} \boldsymbol{S}}
\end{array}\right]=\left[\begin{array}{c}
0 \\
0 \\
\frac{I}{m l^{2}+I} \\
-\frac{m l^{2}}{m l^{2}+1}
\end{array}\right] .
\end{aligned}
$$


Following Eq. (7), the approximate state at $t[\mathrm{~s}], \boldsymbol{x}(t)$, can be analytically derived as

$$
\boldsymbol{x}(t)=\mathrm{e}^{\boldsymbol{A} t} \boldsymbol{x}(0)+\int_{0}^{t} \mathrm{e}^{\boldsymbol{A}(t-\tau)} \boldsymbol{B} v(\tau) \mathrm{d} \tau=\left[\begin{array}{c}
\theta_{1}(t) \\
\theta_{2}(t) \\
\dot{\theta}_{1}(t) \\
\dot{\theta}_{2}(t)
\end{array}\right],
$$

and the control output at $t[\mathrm{~s}]$ is then obtained as

$$
y(t)=\theta_{1}(t)-\theta_{2}(t)=-\frac{2 \pi\left(2 \pi \dot{\theta}_{2}(0)-A_{m} T\right) t+A_{m} T^{2} \sin \left(\frac{2 \pi t}{T}\right)}{4 \pi^{2}} .
$$

The target boundary condition to be met then becomes

$$
\boldsymbol{x}(T)=\left[\begin{array}{c}
\theta_{1}(T) \\
\theta_{2}(T) \\
\dot{\theta}_{1}(T) \\
\dot{\theta}_{2}(T)
\end{array}\right]=\left[\begin{array}{c}
\frac{\alpha}{2} \\
\frac{\alpha}{2} \\
0 \\
\dot{\theta}_{2}(0)
\end{array}\right] .
$$

By solving $\theta_{1}(T)=\frac{\alpha}{2}$, we obtain

$$
A_{m}=\frac{\alpha \hat{\omega}\left(4 \pi^{2}\left(m l^{2}+I\right)+m g l T^{2}\right)}{4 \pi I T} \operatorname{coth}\left(\frac{\hat{\omega} T}{2}\right), \quad \hat{\omega}:=\sqrt{\frac{m g l}{m l^{2}+I}} .
$$

By solving $\theta_{2}(T)=\frac{\alpha}{2}$, we obtain

$$
\dot{\theta}_{2}(0)=\frac{\alpha \hat{\omega}\left(4 \pi^{2}\left(m l^{2}+I\right)+m g l T^{2}\right)}{8 \pi^{2} I} \operatorname{coth}\left(\frac{\hat{\omega} T}{2}\right) .
$$

By substituting the $A_{m}$ of Eq. (14) and $\dot{\theta}_{2}(0)$ of Eq. (15) into the $y(t)$ of Eq. (12), we obtain

$$
y(t)=-\frac{A_{m} T^{2}}{4 \pi^{2}} \sin \left(\frac{2 \pi t}{T}\right)=-\frac{T^{2}}{4 \pi^{2}} v(t) .
$$

We can understand that the relative joint angle also becomes a simple sinusoidal wave. According to the above method, in this case the resultant motions of the stance leg and reaction wheel satisfy the conditions necessary for stealth walking.

\subsection{Numerical simulations}

We conduct a numerical simulation of the linearized URW model. The physical, control and target system parameters are listed in Table 1 . The target step period, $T$, is chosen as a little large to maintain the vertical ground reaction force positive during motion. The URW started from the following initial state:

$$
\boldsymbol{\theta}(0)=\left[\begin{array}{c}
-\frac{\alpha}{2} \\
-\frac{\alpha}{2}
\end{array}\right], \quad \dot{\boldsymbol{\theta}}(0)=\left[\begin{array}{c}
0 \\
\dot{\theta}_{2}(0)
\end{array}\right],
$$

where $\dot{\theta}_{2}(0)$ is the target initial values of the linearized model determined by Eq. (15). Fig. 2 shows the simulation results of high-speed stealth walking of the linearized URW. Here, (a) is the angular positions, (b) the angular velocities, (c) the control input, and (d) the specific resistance (SR). From (c), we can understand that the control load is monotonically reduced with respect to the increase of $k$. The energy efficiency can be evaluated in terms of $\mathrm{SR}$ that is defined as $\mathrm{SR}=p / m g V[-]$ where

$$
p:=\frac{1}{T} \int_{0}^{T}|\dot{y} u| \mathrm{d} t, \quad V:=\frac{2 l \sin \frac{\alpha}{2}}{T} .
$$




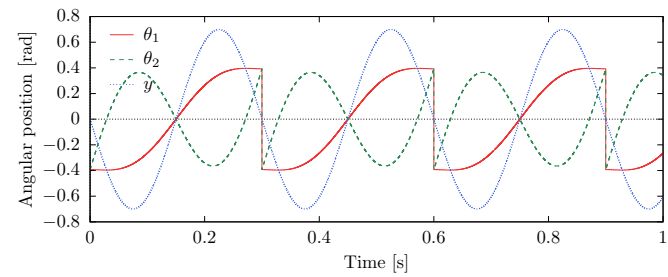

(a) Angular position

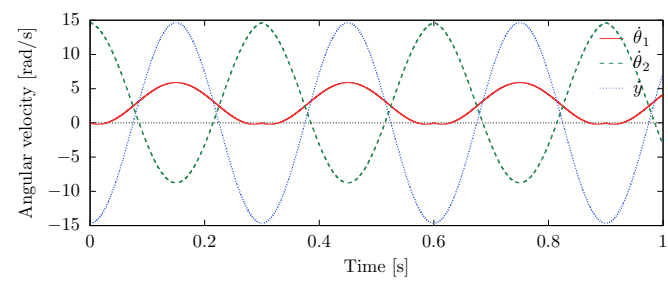

(b) Angular velocity

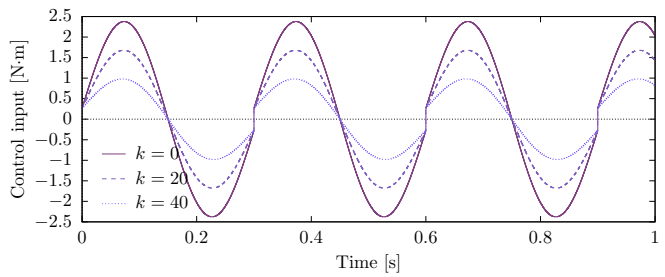

(c) Control input

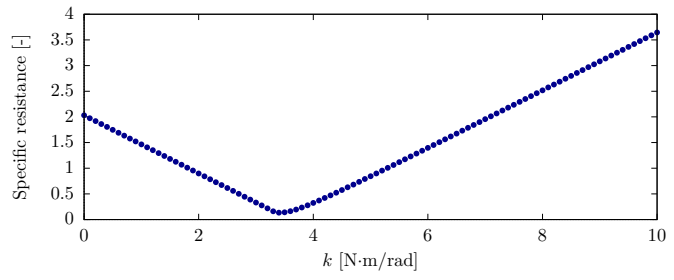

(d) Specific resistance

Fig. 2. Simulation results of high-speed stealth walking of linearized model

Table 1. Physical and control parameters for linearized model.

\begin{tabular}{|c|c|c|c|c|c|}
\hline$m\left(=m_{1}+m_{2}\right)$ & 2.3 & $\mathrm{~kg}$ & $T$ & 0.3 & $\mathrm{~S}$ \\
\hline$I$ & 0.01 & $\mathrm{~kg} \cdot \mathrm{m}^{2}$ & $\dot{\theta}_{2}(0)$ & 14.6483 & $\mathrm{rad} / \mathrm{s}$ \\
\hline$l$ & 0.1 & $\mathrm{~m}$ & $A_{m}$ & 306.793 & $\mathrm{rad} / \mathrm{s}^{2}$ \\
\hline
\end{tabular}

Fig. 2 (d) shows the analysis result of SR versus $k$ in the linearized model, and we can see that the $\mathrm{SR}$ is minimized at $k=3.4[\mathrm{~N} \cdot \mathrm{m} / \mathrm{rad}]$. In the following, we discuss the optimal condition briefly. The input power can be arranged to

$$
\dot{y} u=\left(k-\frac{4 \pi^{2} m l^{2} I}{T^{2}\left(m l^{2}+I\right)}\right) y \dot{y}-\hat{\omega}^{2} \theta_{1} \dot{y} .
$$

This is a linear function of $k$, and it can be expected that the SR is increased or decreased proportional to $k$. The SR is changed only with the first term of Eq. (19), and it can become zero where $k=\frac{4 \pi^{2} m l^{2} I}{T^{2}\left(m l^{2}+I\right)}=3.05725[\mathrm{~N} \cdot \mathrm{m} / \mathrm{rad}]$. This value is a little smaller than the optimal $k$, but is useful to estimate it. More detailed analysis is left as a future work.

\section{Extension to Nonlinear Model}

\subsection{Numerical procedures for identification of system parameters}

As discussed in Refs. 5,6,10-12, the only problem in extension to the nonlinear model is to identify the target initial state exactly through some numerical procedures while referring the approximate target initial state of the linearized model. In this paper, we use a bisection method again. According to the proposed method, both the stance leg and reaction wheel behave as zero dynamics. As discussed in Ref. 6, however, we can identify their target values separately.

First, we execute a numerical procedure to identify the target step period, $T$, to generate a space-time symmetric motion of the stance leg following Algorithm 4.1. Second, we execute another numerical procedure to identify the target initial angular velocity of the reaction wheel, $\dot{\theta}_{2}(0)$, so that it is identical to $\dot{\theta}_{2}(T)$ following Algorithm 4.2. Without going into detail, in both cases, the error norms reached and converged to the minimum value about in the first fifty steps. 

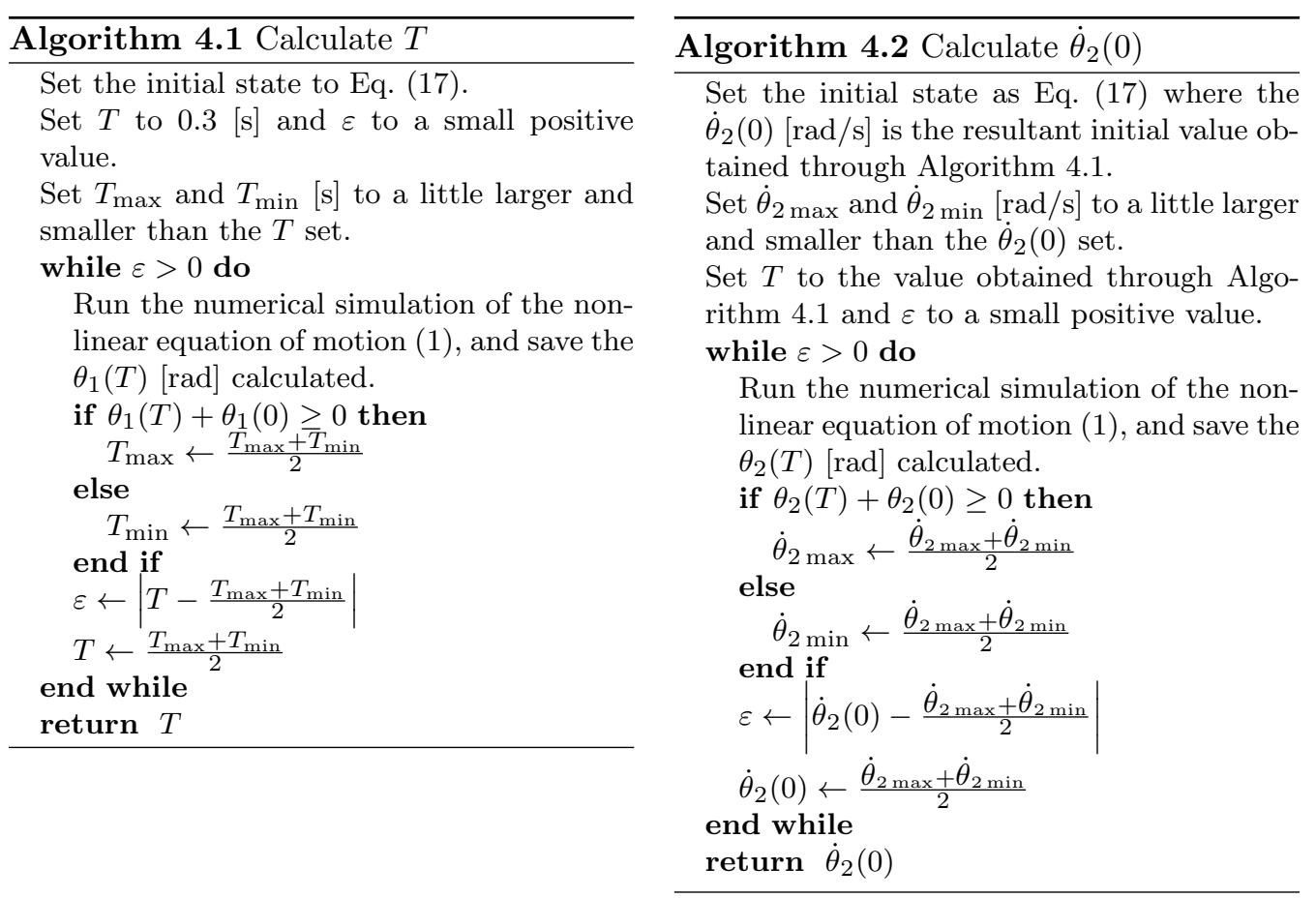

\subsection{Numerical simulations}

Through the above procedures, the target values of the step period and initial angular velocity of the reaction wheel were identified as $T=0.297466[\mathrm{~s}]$ and $\dot{\theta}_{2}(0)=14.5244$ $[\mathrm{rad} / \mathrm{s}]$.

Fig. 3 shows the simulation results of high-speed stealth walking of the nonlinear URW model. We can see that the generated walking motion is similar to that of the linearized model in Fig. 2. The analysis result of the SR versus $k$ in this case is omitted because it is similar to Fig. 2 (d). The minimum SR was 0.129221 [-] where $k=3.5[\mathrm{~N} \cdot \mathrm{m} / \mathrm{rad}]$. From Fig. 3 (d), we can see that the vertical ground reaction force is maintained positive during motion. This condition, however, cannot be met if the target step period $T$ is too short. The lower limit is discussed in the following.

\subsection{Derivation of approximate minimum control period}

The vertical ground reaction force, $F_{z}[\mathrm{~N}]$, is specified as

$$
F_{z}=m \cos \theta_{1}\left(g \cos \theta_{1}-l \dot{\theta}_{1}^{2}\right)-\frac{(u-k y) \sin \theta_{1}}{l} .
$$

Without going into detail, the $F_{z}$ is minimized at mid-stance, $t=\frac{T}{2}[\mathrm{~s}]$. This is strongly supported by Fig. 3(d). The necessary condition then becomes

$$
F_{z}\left(\frac{T}{2}\right)=m g-m \dot{\theta}_{1}\left(\frac{T}{2}\right)^{2} \geq 0 .
$$

This inequality can be arranged and solved for $T$ by using the $\dot{\theta}_{1}\left(\frac{T}{2}\right)$ driven by using the linearized model approximately. The minimum value of $T$ can also be solved accordingly as follows.

$$
\text { Eq. }(21) \Longleftrightarrow \dot{\theta}_{1}\left(\frac{T}{2}\right)=\frac{\alpha \hat{\omega}}{2} \operatorname{coth}\left(\frac{\hat{\omega} T}{4}\right) \leq \omega \Longleftrightarrow T \geq \frac{4}{\hat{\omega}} \tanh ^{-1}\left(\frac{\alpha \hat{\omega}}{2 \omega}\right)=: T_{\min }
$$

The $T_{\min }$ for the simulation model was $0.164673[\mathrm{~s}]$, and the values of $T$ used in the above simulations satisfy the condition of Eq. (22). 


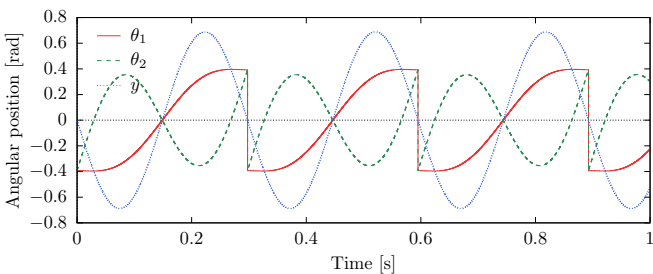

(a) Angular position

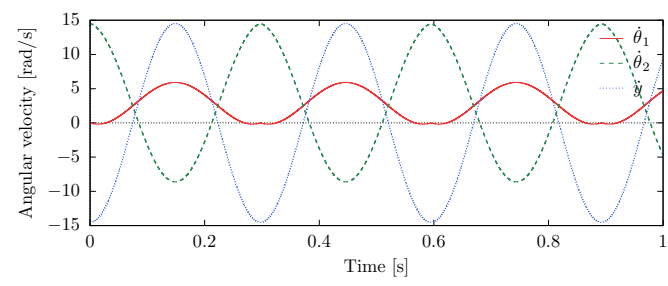

(b) Angular velocity

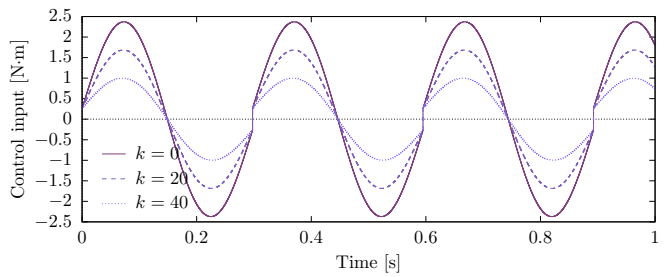

(c) Control input

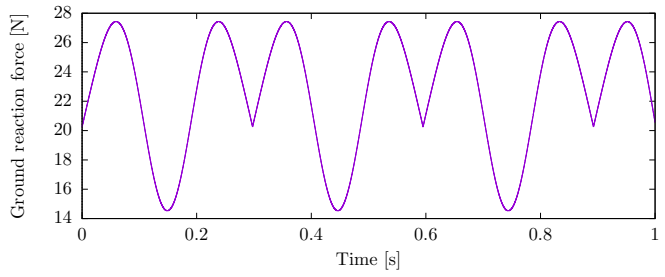

(d) Vertical ground reaction force

Fig. 3. Simulation results of high-speed stealth walking of nonlinear model

\section{Experimental Results}

To verify the validity of the results numerically obtained, we conducted experimental verification using the experimental machine shown in Fig. 1. The main physical parameters of it were the same as those of the mathematical model, and the elastic coefficient is $k=9.5$ $[\mathrm{N} \cdot \mathrm{m} / \mathrm{rad}]$. Fig. 4 shows the desired time trajectory for (a) the angular position, and (b) for the angular velocity of the EC motor. As previously described, the desired time trajectories were designed as sinusoidal waves, and were adjusted several times while referring the simulation results. To reduce the motor load at start and stop, the initial and final trajectories were interpolated by a cubic function. The parameters for $v$ were $A_{m}=1.9548[\mathrm{rad}]$ and $T=0.373832[\mathrm{~s}]$. Fig. 5 shows the snapshots of experimental walking where contours were extracted every second. In the experiment, it was not possible to achieve continuous stealth walking due to the error of the initial angular position and angular velocity, but about 3 steps were successfully continued. For confirmation of successful stealth walking, it was planned to make a judgment from the data of the measured angular position and angular velocity, but the data was inaccurate due to the influence of electromagnetic noise generated from the EC motor. We then judged successful stealth walking based on the collision noise and images of the experimental walking. The basic experiments suggested the possibility that continuous stealth walking could be achieved by setting the initial state more appropriately.

\section{Conclusion and Future Work}

In this paper, we proposed a realistic method for achieving stealth walking of the URW, and investigated the validity through numerical simulations and experiments. Numerical

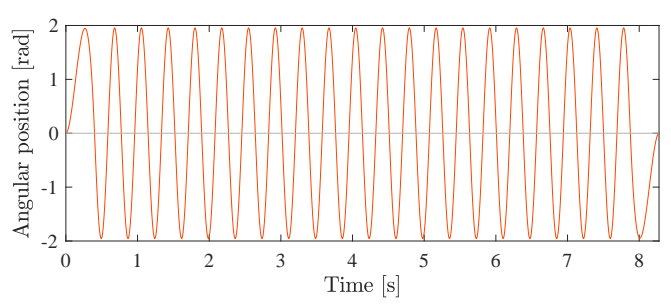

(a) Angular position

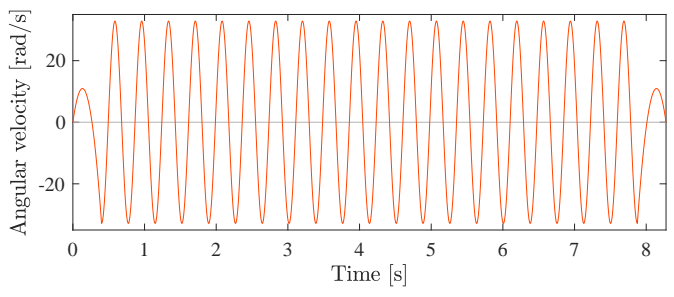

(b) Angular velocity

Fig. 4. Desired time trajectories for angular position and angular velocity in experimental stealth walking 


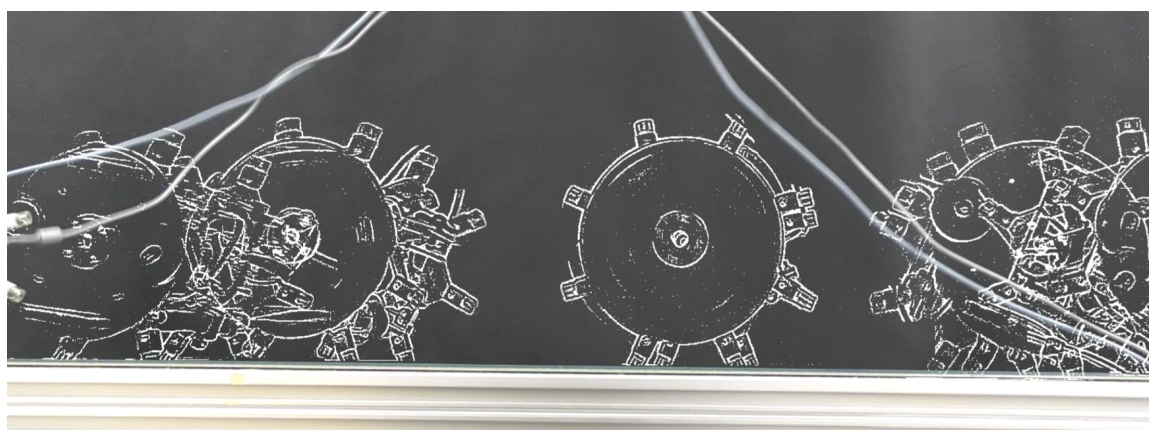

Fig. 5. Snapshots of experimental stealth walking

simulations showed that a stealth walking gait can be successfully generated according to the method; the resultant motions of the stance leg and reaction wheel behaving as zero dynamics could achieve the necessary condition for stealth walking. It was also clarified that the effect of an elastic element can significantly improve the energy efficiency by reducing the load of actuation.

The basic experiments showed that a nearly stealth walking gait can be generated according to the proposed method. Redesigning the data acquisition circuit and development of a catapult for appropriately starting the robot are left as future tasks.

\section{Acknowledgments}

This research was partially supported by SHIBUYA SCIENCE CULTURE AND SPORTS FOUNDATION.

\section{References}

1. E. R. Westervelt, J. W. Grizzle, C. Chevallereau, J. H. Choi and B. Morris, Feedback Control of Dynamic Bipedal Robot Locomotion, CRC Press, 2007.

2. M. Rostami and G. Bessonnet, "Impactless sagittal gait of a biped robot during the single support phase," Proc. of the IEEE Int. Conf. on Robotics and Automation, pp. 1385-1391, 1998.

3. W. Blajer and W. Schiehlen, "Walking without impacts as a motion/force control problem," J. of Dynamic Systems, Measurement, and Control, Vol. 114, Issue 4, pp. 660-665, 1992.

4. L. Gong and W. Schiehlen, "Impactless biped walking on a slope," Theoretical and Applied Mechanics Letters, Vol. 3, Issue 1, 013002, 2013.

5. F. Asano, "Stealth walking with reduced double-limb support phase and its extension to careful legged locomotion," Multibody System Dynamics, Vol. 44, Issue 4, pp. 421-447, 2018.

6. F. Asano and S. Kobayashi, "Generation of strict stealth walking gait using upper body and reaction wheel," Proc. of the 11th IFAC Symp. on Nonlinear Control Systems (NOLCOS), pp. 400-405, 2019.

7. F. Asano, "Efficiency and optimality of two-period limit cycle walking," Advanced Robotics, Vol. 26, Issues 1-2, pp. 155-176, 2012.

8. F. Asano, "Non-powered stealth walking approach to generation of passive dynamic gait on horizontal plane," Proc. of the 12th IFAC Symp. on Robot Control (SYROCO), 002, 2018.

9. F. Asano and X. Xiao, "Output deadbeat control approaches to fast convergent gait generation of underactuated spoked walker," Proc. of the IEEE/SICE Int. Symp. on System Integration (SII), pp. 265-270, 2012.

10. M. W. Gomes and A. Ruina, "Walking model with no energy cost," Physical Review E, Vol. 83, Issue 3, 032901, 2011.

11. T. Chyou, G. F. Liddell and M. G. Paulin, "An upper-body can improve the stability and efficiency of passive dynamic walking," J. of Theoretical Biology, Vol. 285, Issue 1, pp. 126$135,2011$.

12. A. M. Formalskii, "Ballistic walking design via impulsive control," J. of Aerospace Engineering, Vol. 23, Issue 2, pp. 129-138, 2010. 